\title{
Prosthetic Joint Infection Microorganism Pattern And Risk Factor Profile: A Single Center Study
}

Fahreza Hilmy, Yoshi Pratama Djaja , Anggaditya Putra , Jamot Silitonga , Ludwig Andribert Pontoh

Department of Orthopaedic and Traumatology Fatmawati General Hospital, Jakarta, Indonesia

\begin{abstract}
Introduction: Prosthetic joint infection (PJI) is a serious complication especially following arthroplasty surgery. The outcomes of these cases are affected by the pattern of infection, causative microorganism and antibiotic resistance. This study was aimed to evaluate the prevalence of PJI, distribution of causative microorganism, antibiotic resistance, and risk factor profiling.

Methods: A retrospective review was performed by arthroplasty registry evaluation from 2008-2018, followed by medical record review and patient interview. Distribution of causative microorganisms, antibiotic resistance patterns, and onset of infection was extracted. Risk factor evaluation was performed by assessing preoperative (age, body mass index (BMI), comorbidities specifically type 2 diabetes) and perioperative parameters (duration of surgery, number of previous surgeries, interval between those surgeries).
\end{abstract}

Results: Seventeen patients were diagnosed with PJI ( 13 hip and 4 knee), with the prevalence of $1.56 \%$ and $1.77 \%$ respectively. The most common causative organism was Staphylococcus aureus and Escherichia coli with multiple antibiotic resistance patterns. These infections mostly occurred in patients with the age of $40-60$ years, and $\mathrm{BMI}>$ $30 \mathrm{~kg} / \mathrm{m} 2$. Perioperative risk factor was duration of surgery more than 3 hours, have undergone more than 3 surgeries with an average surgical interval of 2 months.

Conclusions: The prevalence of PJI in this series was $1.56 \%$ in hip and $1.77 \%$ in knee. Risk factor profile showed that most cases have high BMI, prolonged duration of surgery, and high number of previous surgeries within a short interval.

Keywords: Prosthetic Joint Infection, Microorganism, Arthroplasty, Hip Knee, Risk Factor

Level of Evidence: IV

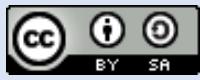

This is an open access article under the CC-BY-SA license.

Article history

Submitted: June $23^{\text {th }}, 2020$

Revise : July $5^{\text {th }}, 2020$

Accepted : July $7^{\text {th }}, 2020$
Corresponding Author: Yoshi Pratama Djaja, MD. Department of Orthopaedic and Traumatology Fatmawati General Hospital, Jakarta, Indonesia. email: yoshiprtamadjaja@gmail.com 


\section{Introduction}

Patients with severe hip and knee pathology typically underwent joint arthroplasty to restore function and improving their quality of life. ${ }^{1}$ One of the most challenging complications of joint arthroplasty is periprosthetic joint infection (PJI), which occurs in 0.78 to $2 \%$ of cases. ${ }^{2,3}$ The risk of $\mathrm{PJl}$ is known to increase in elderly patients, patients with morbid obesity (BMI $>30 \mathrm{~kg} / \mathrm{m} 2$ ), underweight (BMI <18.5), pre-operative health conditions (such as immunity, diabetes, smoking), bilateral arthroplasty, allogenic transfusion, myocardial infarction, urinary tract infection, postoperative atrial fibrillation, and longer hospitalization. ${ }^{1,3} \mathrm{~A}$ study in the UK revealed that the rates of $\mathrm{PJI}$ in both underweight and obese patients doubles the rates of normal patients. ${ }^{3}$ The morbidities caused by PJI may span from mild discomfort, pain, impaired mobility, psychological detriments, extended hospitalization, to longterm disability or even death especially in the frail and elderly population. ${ }^{4}$ The risk of mortality in patients who underwent joint arthroplasty surgery is increased by 2.5-7 fold if the patients developed PJI. ${ }^{5}$ In addition, PJI is also recognized as a leading cause for revision surgery accounting for up to 23$25 \%$ of revisions in the USA and UK. 3,6

The causative pathogens in PJI are mostly consisted of gram positive bacteria, which account for $60.1 \%$ of knee arthroplasty. The most frequent organism is methicillin-resistant Staphylococcus aureus, followed by methicillin-sensitive Staphylococcus aureus and Staphylococcus epidermidis. ${ }^{7}$ Common gram negative pathogens found in PJI are Escherichia coli and Klebsiella pneumoniae. It should be noted that some patients may develop polymicrobial infection, albeit in rare cases. ${ }^{1,7}$ The distribution of pathogens also follow certain predilections depending on geographical areas and surgical sites. $^{8}$ For instance, anaerobic pathogens comprise of only $3-6 \%$ of PJIs but Propionibacterium acnes was found in nearly $25 \%$ of shoulder arthroplasty infections. ${ }^{9,10}$ Understanding the common pathogens associated with a given $\mathrm{PJI}$ is important as the type of pathogens in a given $\mathrm{PJI}$ is known to influence clinical outcomes and prognoses. ${ }^{11}$ One study showed that PJls caused by methicillin-resistant Staphylococcus aureus are associated with a higher risk of surgical failure and poor prognoses. ${ }^{11}$ Currently, there are limited studies regarding PJIs in Indonesia, necessitating more studies in this field to provide better information to clinicians. This study aims to describe the distribution of infection and identifying the risk factors associated with PJI following hip and knee arthroplasty in our center.

\section{Methods}

\section{Patients Selection}

A retrospective descriptive study was conducted by collecting data from our institutional arthroplasty registry. The study protocol was approved by our institutional review board and informed consent was waived since the patients were not recalled for this study. We identified 1060 arthroplasty surgeries (834 hip and 226 knee) were performed between January 2008 and December 2018. From this registry, all PJI cases was consecutively enrolled. The inclusion criteria of this study were patients diagnosed with hip or knee PJI based on the diagnostic criteria for PJI. (Table 1$)^{12}$ The exclusion criteria were as follows: incomplete microbiology data, refusal to participate or unable to be contacted. Medical record review and phone call interview was performed to obtain detailed information of the patients.

\section{Data Extraction and Analysis}

The data extracted from medical records were: onset of infection to determine the classification of PJI (acute or chronic), microorganism involved, and antibiotic resistance pattern. Patient demographics data were also obtained from the medical record, such as age, gender, body mass index (BMI) and comorbidities (specifically type 2 Diabetes Mellitus). Furthermore, perioperative data (number of previous surgeries, interval between surgeries and the duration of latest surgery before the PJI was diagnosed) was also obtained from the medical record or by interview if the data was not found in the record. Previous surgery was defined as the surgeries on the hip or knee that was performed before the infection occurred (it may span from an open reduction internal fixation surgery, arthroplasty or even earlier revision arthroplasty due to various reasons). 
The type of PJI was determined based on onset of PJI after surgery, with acute PJls occurring <3 months after surgery. ${ }^{13}$ Antibiotic pattern resistance is identified using the criteria by Magiorakos et al (Table 2). ${ }^{14}$ Data analysis was performed using SPSS version 21. The data obtained would be evaluated for their frequencies, mean values, and standard deviation, if the data distribution were normal according to the Shapiro-Wilk normality test, with $p>0.05$. If not, median and minimal to maximal data were displayed.

\section{Results}

\section{Patient Demographics}

A total of 17 cases of hip PJI (13 cases) and knee PJI cases (4 cases) were included in this study, which made the prevalence of hip and knee $\mathrm{PJI}$ in our institution were $1.56 \%$ and $1.77 \%$, respectively. Patient characteristics are presented in Table 3 and Table 4. The mean age of patients with knee PJI was higher than the hip PJI cases. The mean BMI was comparable between both groups. Number of previous surgeries and duration of latest surgery were higher in patients with hip PJI, whereas the knee PJI patients had longer interval between surgeries. Nine patients (52.9\%) also had diabetes mellitus as their comorbidity.

\section{Type of PJ}

Most of hip PJI (9 out of 13) cases have delayed/chronic onset, with a mean onset of 5.69 \pm 3.83 months. Acute hip PJI had the onset of 2.3 \pm 0.96 months; meanwhile the chronic one was averaged $7.22 \pm 3.63$ months.

As for knee PJI, the proportion of acute and chronic PJI was comparable (2 cases in each type), with the mean onset of $3.5 \pm 1.29$ months $(2.5 \pm$ 0.71 in acute and $4.5 \pm 0.71$ in chronic PJI).

\section{Microorganism and Antibiotic Resistance Pattern}

Culture of PJI samples revealed that Gram positive pathogens predominated in the THA group (5 cases). However, there were more Gram-negative pathogens in the TKA group (2 cases). Negative culture results were also found in both hip and knee PJI.
In both hip and knee PJI, MDR pathogens were the most common findings. A smaller number of cases were infected by XDR pathogens. Fortunately, there were no PDR pathogens identified in the cases this study.

\section{Discussion}

$\mathrm{PJl}$ is a common and serious post-operative complication in hip and knee arthroplasty. Existing literature has shown that numerous risk factors associated with PJI. The type of pathogen responsible for the infection is also known to affect clinical outcomes and prognosis. Recognizing the risk factors that influence the incidence of this complication, as well as the most common type of pathogens culpable may better inform clinicians to take preventive measures. As mentioned before, this study was aimed to describe the distribution of infection and identified the risk factors associated.

The prevalence of our study was comparable with other studies. ${ }^{2,3}$ The annual incidence rate of PJI ranged at about 1.99-2.18\% for the hip and $2.05-2.18 \%$ for the knee, with there being slightly higher percentage for the knee, which is similar to our findings. ${ }^{15}$ The number the hip PJI cases were larger than the knee, which was caused by the inclusion of both hemiarthroplasty and total hip replacement cases in our series. However, the exact prevalence of PJI in our series was unable to be extracted. We included all PJI cases that were treated in our institution regardless of where the index surgery was performed.

Risk factor profile was identified in the demographic data. Older age, higher BMI, increased number of surgeries and shorter interval between them has been proven as the risk factor of PJI. ${ }^{15,16,17}$ Previous studies has shown that in older patients, $\mathrm{PJI}$ is more frequent and occurred earlier than in younger people. ${ }^{16}$ This finding is concurrent with our series, which showed that a majority of our patients had a relatively advanced age especially in the knee group and these patients also experienced an earlier onset of infection (acute). Interestingly, the average age of hip PJl cases was quite young. The fact, that many hip arthroplasties were actually performed in younger patients (e.g. avascular necrosis, early arthritis due to dysplasia), may be a plausible explanation for our findings. 
$B M I$ is also recognized as another risk factor to PJI, as delineated by the study by Wagner et al which showed that obese patients (BMI $>30 \mathrm{~kg} / \mathrm{m} 2$ ) experienced an increased rate of postoperative infection. ${ }^{17}$ One study by Lubbeke et al asserted several explanations for this correlation, namely the underdosing of antibiotics due to increased body mass, thickness of subcutaneous adipose tissue at site of operation thereby increasing predisposition to infection due to poorer perfusion and lower oxygen tension, enhanced risk of hematoma and higher mechanical tension in wound which may lead to dehisence. ${ }^{18}$ Moreover, obese patients may experience more soft tissue damage and prolonged surgery. ${ }^{18}$ From an immunological perspective, it is established that adipose tissue produces mediators that may potentially impair the immune system. ${ }^{19}$ Most of our PJI cases (12 out of 17 cases) occurred in obese patients with a mean BMI of $30.07 \pm 1.10$ in the hip $P J l$ group and $31.34 \pm 0.14$ in the knee PJl group.

Type II diabetes is a recognized comorbid disease that predisposes a post-operative patient to PJI. A meta-analysis of 14 studies by Zhu et al demonstrated that across eight studies, concurrent type II diabetes proved to be a statistically significant predictor of PJI. ${ }^{20}$ This is consistent with our findings which showed more than half ( 9 out of 17 patients) with PJI also had type II diabetes as a comorbid. The same metaanalysis also identified several surgery-related risk factors for PJI including prolonged surgical duration and previous surgeries. The analysis of the sample studies in this meta-analysis stated that the odds ratio (OR) of prolonged surgical duration and previous surgeries were 2.18 and 3.15 and with $p$ value of 0.001 and 0.003 respectively. ${ }^{20}$. All cases in our series has a history of previous surgery. Among them, 13 out of 17 patients had a surgery in less than 6 months prior to their last procedure. Although there were no data regarding the interval between previous surgeries, we believe shortened interval between the surgeries will increase the risk of infection. Further investigation is needed in this topic.

It is known that the most frequently found microorganisms in $\mathrm{PJI}$ are gram positive organisms, this is thought to be due to their predominance in the normal flora of human skin. ${ }^{21}$ With regards to hip infections, it is thought that the causative pathogens are usually normal flora of the genitals due to their proximity. ${ }^{21}$ Despite the popular notion that most infections are due to the monomicrobial infection of gram positive pathogens, particularly Staphylococcus aureus, one study by Peel et al debunked this finding, with results that state that majority of joint infections comprise of mixed gram positive-negative organisms. $^{22}$

The widespread indiscriminate use of antibiotics in many healthcare facilities has led to the rise of prophylactic failure in post-operative patients. ${ }^{21} \mathrm{~A}$ study by Tornero et al found that methicillin resistance accounted for up to $9 \%$ of PJIs due S. aureus, and $60 \%$ due to $\mathrm{S}$. epidermidis, and fluoroquinolone resistance was made up of $16 \%$ S. aureus and $35 \%$ S. epidermidis infections respectively. ${ }^{23}$ Another study by Martinez et al showed that up to $85 \%$ of E.coli isolated from PJls produced beta lactamases. ${ }^{24}$ It is also important to note that these patterns vary based on the frequency and type of antibiotics abused in different hospitals. ${ }^{21}$ Recognizing the common microorganism patterns in PJI is also important to guide clinicians in forming empirical antibiotic regimens and possibly preventing PJIs entirely.

There are several limitations in our study. Due to its retrospective design, our study is susceptible to recall bias. The number of negative culture results was also relatively high compared to other studies. This may be due to the fact that antibiotic regimens were not halted prior to culture examination, which may decrease the concentration of the causative microorganism in the sample. And as mentioned before, our prevalence may be inaccurate as not all PJI cases in this series were originally treated in our institution. Low number of positive PJI cases was also a limitation of this study. However, considering this was a single center study and the prevalence was proportionate with previous studies, multicenter collaboration was needed to improve the analysis. Despite these limitations, the strength of our study was the involvement of microbiology data and its resistance pattern that was not commonly reported in other similar studies.

\section{Conclusions}

In conclusion, the overall prevalence of $\mathrm{PJI}$ in our study is relatively rare (1.56\% for hip PJI and $1.77 \%$ for knee PJI). Our study population had a predilection for several characteristics known to 
predispose a patient to $\mathrm{PJI}$ including old age, $\mathrm{BM}>30 \mathrm{~kg} / \mathrm{m} 2$, type 2 diabetes, increased duration of surgery, short interval with previous surgery, and a prolonged operative time. Grampositive microbes were more common in PJ patients that underwent hip arthroplasty, whereas gram-negative microbes were more frequent for the knee arthroplasty. Future multicenter collaboration study with bigger sample was needed to obtain better analysis.

\section{Conflict of Interest}

The authors affirm no conflict of interest in this study

\section{Acknowledgement}

None.

\section{References}

1. Pulido L, Ghanem E, Joshi A, Purtill JJ, Parvizi J. Periprosthetic joint infection: the incidence, timing, and predisposing factors. Clin Orthop. $2008 \mathrm{Jul} ; 466(7): 1710-1715$.

2. Zimmerli $W$, Trampuz A, Ochsner PE. Prosthetic-Joint Infections. N Engl J Med. 2004;351(16):1645-1654

3. Lamagni T. Epidemiology and burden of prosthetic joint infections. I Antimicrob Chemother [Internet]. 2014; 69(suppl 1):i510

4. Cahill JL, Shadbolt B, Scarvell JM, Smith PN. Quality of life after infection in total joint replacement. J Orthop Surg (Hong Kong). 2008;16(1):58-65.

5. Coello R, Charlett A, Wilson J, Ward V, Pearson A, Borriello P. Adverse impact of surgical site infections in English hospitals. $J$ Hosp Infect. 2005;60(2):93-103.

6. Clohisy JC, Calvert G, Tull F, McDonald D, Maloney WJ. Reasons for revision hip surgery: a retrospective review. Clin Orthop. 2004;(429):188-92.

7. Wang F-D, Wang Y-P, Chen C-F, Chen H-P. The incidence rate, trend and microbiological aetiology of prosthetic joint infection after total knee arthroplasty: A 13 years' experience from a tertiary medical center in Taiwan. J Microbiol Immunol Infect. 2018; 51(6):717-22.
8. Tsai J-C, Sheng W-H, Lo W-Y, Jiang C-C, Chang S-C. Clinical characteristics, microbiology, and outcomes of prosthetic joint infection in Taiwan. I Microbiol Immunol Infect. 2015;48(2):198-204.

9. Shah NB, Tande AJ, Patel R, Berbari EF. Anaerobic prosthetic joint infection. Anaerobe. 2015;36:1-8.

10. Piper KE, Jacobson MJ, Cofield RH, Sperling JW, Sanchez-Sotelo J, Osmon DR, et al. Microbiologic diagnosis of prosthetic shoulder infection by use of implant sonication. J Clin Microbiol. 2009;47(6):1878-84.

11. Salgado CD, Dash S, Cantey JR, Marculescu CE. Higher risk of failure of methicillinresistant Staphylococcus aureus prosthetic joint infections. Clin Orthop. 2007;461:4853.

12. Enayatollahi MA, Parvizi J. Diagnosis of infected total hip arthroplasty. Hip Int. 2015;25(4):294-300.

13. Aggarwal VK, Rasouli MR, Parvizi J. Periprosthetic joint infection: Current concept. Indian J Orthop. 2013;47(1):10-7.

14. Magiorakos A-P, Srinivasan A, Carey RB, Carmeli Y, Falagas ME, Giske CG, et al. Multidrug-resistant, extensively drugresistant and pandrug-resistant bacteria: an international expert proposal for interim standard definitions for acquired resistance. Clin Microbiol Rev. 2012;18(3):268-81.

15. Tande AJ, Patel R. Prosthetic joint infection. Clin Microbiol Rev. 2014;27(2):302-45.

16. Hsieh PCH, Segers VFM, Davis ME, MacGillivray C, Gannon J, Molkentin JD, et al. Evidence from a genetic fate-mapping study that stem cells refresh adult mammalian cardiomyocytes after injury. Nat Med. 2007;13(8):970-4.

17. Wagner ER, Kamath $A F$, Fruth $K M$, Harmsen WS, Berry DJ. Effect of Body Mass Index on Complications and Reoperations After Total Hip Arthroplasty. J Bone Joint Surg Am. 2016 3;98(3):169-79.

18. Lübbeke $A$, Zingg $M, V u ~ D$, Miozzari $\mathrm{HH}$, Christofilopoulos $\mathrm{P}$, Uçkay I, et al. Body mass and weight thresholds for increased prosthetic joint infection rates after primary total joint arthroplasty. Acta Orthop. 2016;87(2):132-8. 
19. Marti A, Marcos A, Martinez JA. Obesity and immune function relationships. Obes Rev. 2001;2(2):131-40.

20. Zhu Y, Zhang F, Chen W, Liu S, Zhang Q, Zhang Y. Risk factors for periprosthetic joint infection after total joint arthroplasty: a systematic review and meta-analysis. J Hosp Infect. 2015;89(2):82-9.

21. Ortega-Peña $S$, Colín-Castro $C$, HernándezDuran M, López-Jácome E, Franco-Cendejas R. Microbiological characteristics and patterns of resistance in prosthetic joint infections in a referral hospital. Cir Cir Engl Ed. 2015;83(5):371-7

22. Peel TN, Cheng AC, Buising KL, Choong PFM. Microbiological aetiology, epidemiology, and clinical profile of prosthetic joint infections: are current antibiotic prophylaxis guidelines effective? Antimicrob Agents Chemother. 2012;56(5):2386-91.

23. Tornero E, Senneville E, Euba G, Petersdorf $S$, Rodriguez-Pardo D, Lakatos $B$, et al. Characteristics of prosthetic joint infections due to Enterococcus sp. and predictors of failure: a multi-national study. Clin Microbiol Infect. 2014;20(11):1219-24. MartínezPastor JC, Muñoz-Mahamud E, Vilchez F, García-Ramiro S, Bori G, Sierra J, et al. Outcome of acute prosthetic joint infections due to gram-negative bacilli treated with open debridement and retention of the prosthesis. Antimicrob Agents Chemother. 2009;53(11):4772-7. 
Table 1. Diagnostic Criteria for PJ ${ }^{12}$

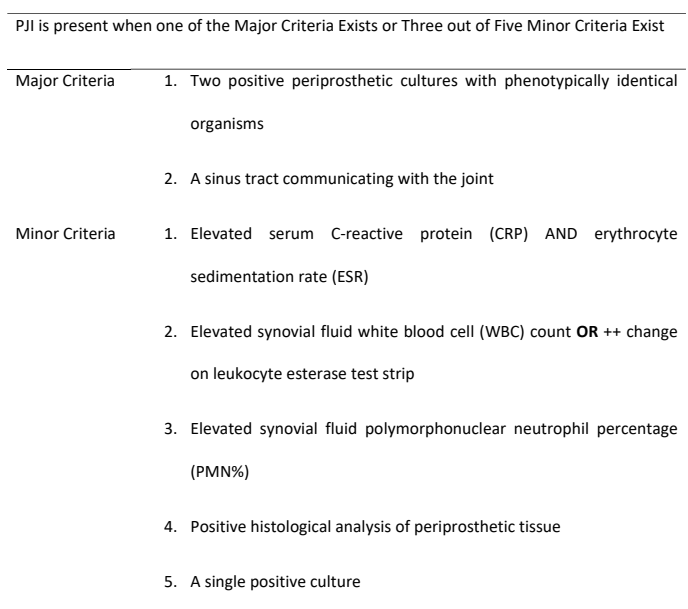

Table 2. Antibiotic Resistance Pattern Criteria

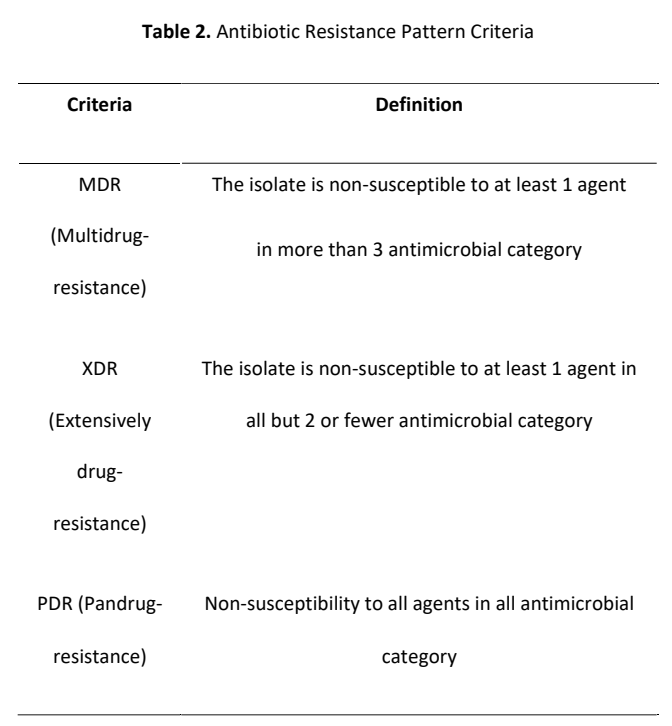

Table 3. Patient Demographics

\begin{tabular}{|c|c|c|}
\hline & $\begin{array}{l}\text { Hip PJI } \\
(n=13)\end{array}$ & $\begin{array}{l}\text { Knee PJI } \\
(n=4)\end{array}$ \\
\hline Age & $48.85 \pm 19.76$ & $71 \pm 13.09$ \\
\hline \multicolumn{3}{|l|}{ Gender } \\
\hline$\square \quad$ Male & 9 & 2 \\
\hline$\square$ Female & 4 & 2 \\
\hline BMI & $30.07 \pm 1.10$ & $31.34 \pm 0.14$ \\
\hline Number of & $3(1-5)$ & $1(1-2)$ \\
\hline \multicolumn{3}{|l|}{ Surgeries } \\
\hline Interval Between Previous & $3.77 \pm 2.17$ & $6.25 \pm 4.03$ \\
\hline two Surgeries (month) & & \\
\hline $\begin{array}{l}\text { Duration of Latest Surgery } \\
\text { (minute) }\end{array}$ & $194 \pm 27.24$ & $145 \pm 46.55$ \\
\hline
\end{tabular}


Table 4. Summary of Patient Data

\begin{tabular}{|c|c|c|c|c|c|c|c|c|c|c|}
\hline Initials & Age & Gender & BMI & Comorbidities & $\begin{array}{c}\text { Number of } \\
\text { Previous } \\
\text { Surgeries }\end{array}$ & $\begin{array}{c}\text { Surgeries } \\
\text { Interval } \\
\text { (Months) }\end{array}$ & $\begin{array}{c}\text { Last Surgery } \\
\text { Duration } \\
\text { (minutes) }\end{array}$ & $\begin{array}{l}\text { Onset of } \\
\text { Infection }\end{array}$ & Microorganism Found & $\begin{array}{c}\text { Antibiotic } \\
\text { Resistance } \\
\text { Pattern }\end{array}$ \\
\hline \multicolumn{11}{|l|}{ Hip PJI } \\
\hline RB & 52 & $\mathrm{~F}$ & 37.91 & Type II diabetes & 3 & 6 & 230 & 14 & Acinetobacter baumanii & XDRO \\
\hline DS & 53 & M & 34.89 & N/A & 4 & 5 & 180 & 6 & Negative & N/A \\
\hline SET & 30 & $\mathrm{~F}$ & 30,42 & N/A & 1 & 0 & 170 & 2 & Staphylococcus epidermidis & MDRO \\
\hline SIR & 25 & M & 30,23 & N/A & 2 & 6 & 180 & 5 & Staphylococcus epidermidis & MDRO \\
\hline AA & 53 & M & 33.56 & Type II diabetes & 3 & 4 & 150 & 12 & Negative & N/A \\
\hline ZA & 64 & $M$ & 26.12 & N/A & 1 & 0 & 170 & 6 & Negative & N/A \\
\hline RS & 89 & $\mathrm{~F}$ & 29,54 & Type II diabetes & 2 & 7 & 180 & 8 & Escherichia coli & MDRO \\
\hline $\mathrm{R}$ & 36 & M & 31,57 & Type II diabetes & 4 & 3 & 230 & 3 & Staphylococcus aureus & MDRO \\
\hline DUD & 24 & $\mathrm{~F}$ & 28,98 & N/A & 3 & 4 & 200 & 4 & Acinetobacter baumanii & MDRO \\
\hline \multicolumn{11}{|l|}{ Knee PJI } \\
\hline IY & 76 & M & 31,24 & Type II diabetes & 2 & 4 & 160 & 2 & Escherichia coli & XDRO \\
\hline ES & 54 & M & 31,45 & N/A & 1 & 3 & 200 & 4 & Pseudomonas Aeruginosa & MDRO \\
\hline RZ & 69 & $\mathrm{~F}$ & 37.46 & N/A & 1 & 12 & 90 & 5 & Negative & N/A \\
\hline $\mathrm{P}$ & 85 & $\mathrm{~F}$ & 34.63 & Type II diabetes & 1 & 6 & 130 & 3 & Staphylococcus epidermidis & MDRO \\
\hline
\end{tabular}

